\title{
La incorporación de la ética ambiental en la planificación y gestión del medio ambiente
}

\author{
The incorporation of environmental ethics in the \\ environmental planning and management
}

\section{Mario Burgui Burgui ${ }^{1}$ \\ Edson Vicente da Silva ${ }^{2}$ \\ Francisco Otavio Landim Neto $^{3}$}

\begin{abstract}
1 Cátedra de Ética Ambiental “Fundación Tatiana Pérez de Guzmán el Bueno”, Universidad de Alcalá (Madrid, España). E-mail: mario.burgui@uah.es 2 Departamento de Geografia, Universidade Federal do Ceará (Brasil. E-mail: cacauceara@gmail.com

3 Departamento de Geografia, Universidade Federal do Amapá. E-mail: otaviotese@gmail.com
\end{abstract}

RESUMEN: En este artículo se expone que las causas últimas de la crisis ambiental, y por tanto también la búsqueda de soluciones, requieren una reflexión profunda más allá de criterios científicos, técnicos o económicos. En esta reflexión la ética ambiental puede ser de gran utilidad, toda vez que la planificación y gestión ambiental dependen en gran medida de nuestra visión de la naturaleza y de qué relación mantengamos con nuestro entorno. Por ello, se plantea la conveniencia de una mayor inclusión de consideraciones éticas en las políticas ambientales y en los distintos niveles de planificación y gestión ambiental.

Palabras clave: Ética ambiental. Medio ambiente. Naturaleza. Planificación ambiental. Gestión ambiental.

ABSTRACT: This paper discusses how the root causes of the environmental crisis, and therefore the solutions needed, require a deep reflection beyond scientific, technical or economic criteria. In this reflection, environmental ethics can be extremely useful, since environmental planning and management depend heavily on our vision of nature and the kind of relationship we have with our environment. Consequently, this paper proposes a greater incorporation of ethical considerations into environmental policies and the various levels of planning and environmental management.

Keywords: Environmental ethics. Environment. Nature. Environmental planning. Environmental management.

Sumario: Introducción - 1 Visión y Gestión del Medio Ambiente - 2 Conservación o Protección de la Naturaleza - 3 Ética y Responsabilidad Ambiental - 4 El Proceso de Inclusión de la Ética Ambiental en la Planificación y Gestión del Entorno - Consideraciones Finales - Referencias

\section{INTRODUCCIÓN}

Cada vez existe un mayor acuerdo en que las verdaderas causas de la crisis ambiental se encuentran más allá de la esfera económica, científica o técnica. Los análisis y diagnósticos realizados desde distintos sectores y disciplinas llegan a la misma conclusión: se trata de una crisis cultural, de valores, del modo en cómo vemos nuestro entorno y cómo nos hemos concebido a nosotros mismos y nuestro papel en el mundo (Pigem, 2006). Según esta perspectiva, la crisis ambiental en toda su extensión constituiría una consecuencia de otra crisis más profunda.

Si se acepta esta premisa, la búsqueda de soluciones a los problemas ambientales (incluyendo el 
cambio climático) debería tener en cuenta no sólo las medidas científico-técnicas más adecuadas con una utilización sostenible de la economía, sino también un cambio profundo en nuestra escala de valores, en el sentido de la palabra progreso y, sobre todo, un compromiso moral a todos los niveles: individual, comunitario, empresarial, gubernamental, etcétera (Chuvieco, 2016).

Actualmente, en esta reflexión sobre las raíces de la crisis ambiental se está tomando con mayor consideración la voz de algunos sectores que hasta épocas recientes apenas habían tenido protagonismo (Elcacho, 2015) ${ }^{1}$, como la filosofía, la religión o el arte, cuyas aportaciones suponen un enriquecimiento hacia la correcta comprensión de los dilemas ambientales y del objetivo final que se debe buscar con la gestión del medio ambiente.

La ética siempre se ha considerado una guía imprescindible para la valoración de nuestras acciones, un referente sobre qué está bien o qué está mal. Hasta hace pocas décadas, la valoración ética se encontraba restringida exclusivamente a la esfera humana, incluso aunque el conflicto afectase a otros seres vivos o a determinado espacio más o menos natural. En este marco de pensamiento antropocéntrico, se consideraba que sólo el ser humano era digno de consideración moral (entre otras cosas, porque sólo él tenía valor intrínseco).

Sin embargo, desde las últimas décadas del siglo XX, se viene proponiendo extender la frontera ética también hacia otros seres vivos que algunos pensadores estiman con valor intrínseco (biocentrismo) o incluso hacia el conjunto de elementos que forman un ecosistema (ecocentrismo). De este modo se han ido generando distintas corrientes dentro de lo que se ha denominado académicamente ética ambiental o ecoética, orientada a la reflexión sobre la dimensión moral de los problemas ambientales.

La ética ambiental comienza a considerarse oficialmente como disciplina en los años 1970 del pasado siglo, coincidiendo con la celebración del primer congreso sobre filosofía ambiental en la Universidad de Georgia (USA) en 1971, la aparición de un notable número de monografías relevantes sobre esta temática y de las primeras revistas académicas dedicadas específicamente a ella, como es el caso de Environmental Ethics (Chuvieco \& Martín, 2015) ${ }^{2}$.

Se podría decir que hay tantas éticas ambientales como sistemas éticos, pero en cualquier caso, el juicio ético debería ser un componente esencial de nuestra actuación en el medio, ayudando en la resolución de los dilemas que esa actuación provoca.

\section{VISIÓN Y GESTIÓN DEL MEDIO AMBIENTE}

Uno de los focos de atención de la ética ambiental es la discusión sobre el valor de la naturaleza. ¿Tiene valor en sí misma (valor intrínseco), o su valor es meramente instrumental, al servicio de los intereses del ser humano? (Velayos, 1996) ${ }^{3}$. En función de cómo se responda a esta pregunta, si existe coherencia

\footnotetext{
${ }^{1}$ Aunque la encíclica Laudato Si' del Papa Francisco parece estar marcando un punto de inflexión, también líderes religiosos de otras grandes religiones se han venido involucrando recientemente en la lucha contra el cambio climático. Elcacho, Joaquim, "Los líderes budistas también reclaman un acuerdo contra el cambio climático", La Vanguardia, 2015 (http://www.lavanguardia.com/natural/20151030/54438468358/lideres-budistastambien-reclaman-un-acuerdo-mundial-contra-el-cambio-climatico.html).

2 Entre las numerosas publicaciones, se pueden destacar: "Should Trees Have Standing" de C.D. Stone (1971), el informe "Los límites del crecimiento" en 1972 (encargado por el Club de Roma), "Lo pequeño es hermoso" (E.F. Schumacher, 1973), "The shallow and the deep, de Arne Naess (1973), "Man's responsibility on Nature" (J. Passmore, 1974), o "Philosophy and Environmental Crisis" (W.T. Blackstone, 1974).
}

${ }^{3}$ La autora trata, entre otros, el tema del valor intrínseco de la naturaleza, y lleva a cabo un interesante estudio 
entre pensamiento y acción, las consecuencias prácticas en la actuación sobre el medio serán muy distintas.

Esto es, dependiendo de cómo se entienda el papel del ser humano en relación con la naturaleza, y según los objetivos que se consideren prioritarios, las medidas de gestión ambiental pueden variar sustancialmente. Simplificando mucho ${ }^{4}$, un enfoque más antropocéntrico daría prioridad a los beneficios inmediatos para las personas (como un uso recreativo intenso de un área protegida o una mayor inclusión de actividades económicas dentro de su espacio); mientras que los enfoques más biocéntricos o ecocéntricos priorizarían la conservación de las especies y los ecosistemas, por ejemplo restringiendo el uso público en tiempo y espacio si se estima que puede interferir con el ciclo reproductor de ciertos animales, prohibiendo la caza, o incluso suprimiendo completamente la presencia humana (como ha ocurrido en algunos países con la expulsión de pueblos indígenas de espacios protegidos) ${ }^{5}$.

Respecto a la ejecución de un proyecto concreto que suponga una intervención sobre el paisaje (como la construcción de una carretera) si se sostiene una perspectiva que se suele denominar antropocentrismo fuerte, no se vería necesaria ninguna medida de compensación. Desde un antropocentrismo débil se estimaría suficiente para reparar el daño causado una plantación de mismas especies en una superficie similar a la intervenida. Sin embargo, desde un punto de vista más biocéntrico esa compensación se consideraría insuficiente, ya que la pérdida de diversidad y de información genética, la alteración de hábitats o los cambios en la dinámica funcional del sistema no se repararían con la nueva plantación (Rodríguez, 1999).

Otros ejemplos se pueden encontrar en el agua y los sistemas fluviales, a menudo foco de conflictos ambientales, socioeconómicos y políticos. Tanto es así que ya en 1999 la UNESCO creó una subcomisión sobre la 'Ética del Agua', considerando que las cuestiones éticas relativas al uso de los recursos hídricos son parte integral de los problemas ambientales más importantes (Ten Have, 2010). Así, desde un punto de vista antropo-tecnocéntrico, la depuración de aguas contaminadas se podría ver como la solución a los problemas de contaminación de los ríos, si bien los expertos en sistemas fluviales saben que esto no es así (Entralgo, 2011).

Por otro lado, a menudo se identifica erróneamente el río con el cauce, ignorando que el sistema fluvial llega mucho más lejos e incluye toda la llanura de inundación, los antiguos meandros, la zona hiporreica y la conexión con el acuífero, etcétera (Entralgo, 2011). Ignorar esta realidad provoca distintos problemas en los espacios fluviales, como pueden ser la contaminación directa o indirecta, salinización y sobreexplotación de acuíferos, entre otros (Llamas, 2000).

A su vez, la ocupación del territorio fluvial con infraestructuras y edificios sin tener en cuenta las características de este complejo sistema puede acarrear consecuencias nefastas, debido a las inundaciones de distinta magnitud que acontecen cíclicamente de forma natural. Se plantea así la necesidad de estudios exhaustivos para adecuar la ordenación de usos del suelo a las características de las zonas inundables, con el fin de evitar daños materiales y humanos (Ollero, 2014).

crítico de las principales corrientes dentro de la ética ambiental. También repasa otras fundamentaciones de la ética ambiental más allá de la posesión de valor (las virtudes, el derecho y la justicia ambiental, la deontología, la ética del discurso, etc.

${ }_{4}$ Sin embargo, la realidad nunca es tan simple y existen muchas variantes tanto dentro del antropocentrismo, como de los distintos biocentrismos y ecocentrismos. Véase: Marcos, Alfredo, Ética Ambiental, Valladolid, Universidad de Valladolid, 2001, pp. 61-86, 121-158.

${ }^{5}$ Aunque está demostrado que las políticas de conservación de la naturaleza son mucho más efectivas cuando se involucra en ellas a la gente del lugar. Amend, Thora \& Amend, Stephan (eds.), ¿Espacios sin habitantes?: parques nacionales de América del Sur, Caracas, UICN/Ed. Nueva Sociedad, 1992, 497 p. 
Tratar de 'controlar' los sistemas fluviales con medidas como la canalización de tramos, los dragados, la instalación de escolleras o la regulación de caudales mediante la ampliación de embalses, puede resultar en el traslado del problema a otro lugar o la creación de un conflicto socio-ambiental donde antes no existía (Arrojo, 2010), sin olvidar los efectos negativos en el mismo lugar donde se aplican (eliminación de vegetación autóctona, desaparición de especies, reducción de caudales ecológicos y de sedimentos, disminución generalizada de la dinámica natural del sistema...).

Frente a esa visión antropo-tecnocéntrica de ‘dominio' de la naturaleza, una visión de 'pertenencia y cuidado’ nos llevaría a replantearnos nuestras prioridades y a adaptar la planificación y gestión ambiental a las características del entorno en la medida de lo posible, reduciendo el grado en que es preciso modificarlo.

Aunque éstos son tan sólo unos ejemplos muy simples, sirven para ver que siempre existe una ideología de fondo, una visión del mundo, de la naturaleza y de nosotros mismos, que influye en planificación y la gestión ambiental, aunque generalmente no se reflexiona sobre el ideario en el que éstas se enmarcan. Las metodologías y técnicas utilizadas se encuentran mayoritariamente dentro del paradigma utilitarista dominante, que prima el valor de las consecuencias para ciertos intereses humanos sobre otras dimensiones éticas (como la consecución de la virtud o la ejemplaridad moral), y el cual ha demostrado no ser suficiente para garantizar una conservación íntegra del ambiente (Kwiatkowska, 2010).

\section{CONSERVACIÓN O PROTECCIÓN DE LA NATURALEZA}

Otro ejemplo en cómo nuestra ideología y cosmovisión influyen en la gestión ambiental es la idea de conservación de la naturaleza, la cual ha ido evolucionando a lo largo del tiempo, desde la primacía de una concepción rígida de la protección en la que se pretendía mantener espacios sin ninguna intervención humana, hacia una concepción más abierta que contempla la posibilidad de la utilización y el aprovechamiento racional de los recursos naturales. Estas distintas concepciones vienen de muy atrás, aunque se podría poner como ejemplo de desencuentro relativamente reciente el debate ideológico que protagonizaron John Muir y Gifford Pinchot. Muir consideraba que todos los elementos del medio (bióticos y abióticos) tenían valor en sí mismos, independientemente de su utilidad para el ser humano. Entendía la naturaleza como un todo, con cierto componente místico, y no aceptaba su utilización mercantilista.

Por su parte, Pinchot podría considerarse un precursor del concepto de desarrollo sostenible, al entender la conservación de la naturaleza desde un punto de vista productivista, buscando la mayor utilidad a los recursos naturales para el interés humano, compatibilizándola con su mantenimiento a lo largo del tiempo (Carcavilla et al., 2007). Hoy en día, estas distintas visiones de la naturaleza siguen influyendo en la gestión que llevamos a cabo de nuestro entorno.

Desde el punto de vista de cómo llevar a cabo la conservación (o protección) de la naturaleza a través de la planificación y ordenación territorial tampoco existe un acuerdo sobre cuál es el modelo más deseable. Hay quienes aplauden la figura de los espacios protegidos, pero también están los que ponen en cuestión sus resultados y proponen una conservación global de todo el territorio (Alba, 1997).

Sin embargo, estas dos opciones no son necesariamente incompatibles, dado que es posible llevar a cabo una gestión racional del medio en su conjunto al tiempo que se reservan áreas con una protección especial. Lo que es indispensable es la articulación de todos los modelos de gestión en las distintas áreas que se zonifiquen de forma coordinada y con una visión sistémica, a través de una ordenación territorial que genere una matriz funcional y efectiva ${ }^{6}$.

\footnotetext{
${ }^{6}$ Siempre teniendo en cuenta los objetivos de conservación de las áreas protegidas a la hora de planificar usos en su entorno, comenzando por su zona de influencia-amortiguación. Mateo, José Manuel, Planificación Ambiental, La Habana, Editorial Félix Varela, 2008, 166 p.
} 


\section{3 ÉTICA Y RESPONSABILIDAD AMBIENTAL}

Hasta el momento, y a pesar de que son imprescindibles, ni el diagnóstico científico de la problemática ambiental ni las soluciones técnicas y administrativas han resultado suficientes para revertir las tendencias de degradación de nuestro entorno. Con el fin de avanzar hacia los objetivos de conservación de la naturaleza y lucha contra el cambio climático, y al mismo tiempo evitar conflictos socioambientales como los mencionados, hoy en día es preciso que reorientemos nuestro modelo de desarrollo y, en lo que a la gestión se refiere, incorporemos de forma creciente postulados éticos en las políticas y planes relacionados con el medio ambiente (Ten Have, 2010).

Pero podría plantearse la pregunta de qué tiene que ver en esto la ética, precisamente en un momento en que el término se utiliza tanto en tan gran diversidad de foros que corre el riesgo de degradarse. Aun así, las políticas de gestión ambiental no están al margen de la ética. Una decisión moral se caracteriza por afectar a terceros y resulta que las decisiones que se toman desde los gobiernos siempre afectan a terceros, (más aún en lo que al medio ambiente se refiere) (García-Marzá, 2007). Incluso lo que en la práctica sucede en la mayoría de las democracias -lamentablemente - es que la maquinaria del Estado decide por todos, muchas veces sin tener en cuenta todas las voces. Esta es una de las razones por las cuales las políticas y decisiones que se toman a nivel institucional tienen un carácter moral.

Sin embargo, las instituciones fueron creadas para satisfacer unos fines que, en el caso del Estado, podrían englobarse en el concepto del 'bien común' (un concepto moral donde los haya, por muy ambiguo o abstracto que se antoje). Si la institución no cumple estos fines, pierde su sentido, su esencia: deja de ser útil. Peor aún, incumple las expectativas de la sociedad, a partir del contrato moral establecido, con lo que dicha institución pierde toda credibilidad. A pesar de que la decisión sobre unas determinadas políticas puede ser tomada por un gran número de personas y no una sola, no existe una institución amoral, porque sus decisiones nos afectan a todos.

No es posible dejar de ser moral, ya se trate de instituciones gubernamentales, empresas, asociaciones, etc. (García-Marzá, 2007). De ahí que no baste con justificar una política, plan o proyecto con argumentos técnicos, administrativos, económicos o científicos. Es necesario que la ética se inserte en el carácter de la institución, en su cultura. Su modo de obrar colectivo debe responder antes de nada a criterios éticos.

En referencia al mundo empresarial, algunas de cuyas experiencias pueden resultar ejemplificadoras para la incorporación de la ética a la planificación y gestión ambiental, Keith Davis ya indicaba que "la sociedad concede legitimidad y poder a la empresa. En el largo plazo, aquellos que no usan este poder de un modo que la sociedad considera responsable tienden a perderlo" (García-Marzá, 2007).

Esto mismo puede aplicarse al Estado y sus políticas públicas. La sociedad tiene unas expectativas de acción del Estado. Si éste las cumple, genera confianza. Si las incumple, desconfianza. Ni que decir tiene que esta confianza es más importante que nunca hoy en día, ante la crisis ambiental y el cambio climático.

Las expectativas de la sociedad son obviamente muchas, pudiendo englobarse en económicas (empleo), sociales (seguridad, sanidad, educación) y también ambientales. Estas últimas han entrado a formar parte del juego sólo en las últimas décadas, cuando cada vez está más claro que sin ellas, las anteriores no podrían ni siquiera plantearse (Mateo, 2003).

Y aprovechando nuevamente la comparativa con el mundo empresarial, generalmente se ha creído que en una corporación "la responsabilidad económica, el beneficio, es la categoría básica sobre la que descansa el resto de los niveles o espacios que componen la responsabilidad” (García-Marzá, 2007) (esto es, sin 
beneficios económicos no podría haber responsabilidad ambiental o social) ${ }^{7}$. En el Estado, es la responsabilidad ambiental y su desarrollo en una planificación y gestión racional de los recursos naturales la que puede garantizar el resto de los espacios de responsabilidad, como el social y el económico.

\section{EL PROCESO DE INCLUSIÓN DE LA ÉTICA AMBIENTAL EN LA PLANIFICACIÓN Y GESTIÓN DEL ENTORNO}

Para que la ética forme parte del carácter de la institución y por tanto de sus políticas y acciones, en primer lugar, se debe cumplir algo muy básico: la participación de todos los actores posibles, ya que todos son afectados por las políticas. Se trataría de garantizar un procedimiento justo y ético desde una perspectiva dialógica: buscar el diálogo y el acuerdo -o consenso- como algo ineludible (García-Marzá, 2007).

Y lo deseable es que el proceso vaya de abajo a arriba, desde la población hacia los gestores gubernamentales (mediante las organizaciones más adecuadas). La gestión ambiental no puede ejecutarse unilateralmente desde un sector - ya sea éste el estatal - o no tendría legitimidad, aunque se adorne de procesos pseudo-participativos (que son sólo informativos la mayoría de las veces). Por otro lado, no hay que olvidar que la responsabilidad ambiental es compartida siempre, ya que el Estado, aunque sea el garante de los derechos y principal responsable de la gestión, nunca es el único responsable. Con una verdadera participación, se garantiza que ni la culpa ni los logros queden sobre uno sólo de los actores.

En definitiva, García-Marzá (2007) resume todo esto en el siguiente principio ético para la gestión de las instituciones: "Una institución es responsable cuando las decisiones, acciones y políticas que adopta, así como las consecuencias y efectos de las mismas respecto a los intereses en juego, pudieran ser aceptadas por todos los implicados y/o afectados presentes y futuros en un diálogo abierto en condiciones simétricas de participación".

Pero además hay que tener en cuenta que la participación pública tiene una serie de ventajas de cara a la conservación ambiental, como por ejemplo (Perero Van Hove, 2002):

Eficacia de las soluciones y mejor cuidado del entorno. Y es que las acciones a ejecutarse en un determinado lugar se van a ajustar mejor a las necesidades de sus habitantes si es de ellos de quienes parte la solicitud de tal acción. Unos ciudadanos que han participado en la toma de decisiones sobre las acciones a llevarse a cabo, las entienden como suyas.

Colaboración con la Administración. Lo anterior prepara el terreno para lograr una mayor colaboración entre la Administración y la sociedad. Y a la postre, unos ciudadanos satisfechos con determinada acción colaborarán en su mantenimiento y cuidado.

Cambio de actitudes. Esta colaboración entre sectores y la participación de los ciudadanos en la mejora de su entorno propicia un cambio de actitudes, que redunda en beneficios para el medio ambiente y también para el sujeto de la acción.

Comprender lo global. Asimismo, este cambio de actitudes prepara al ciudadano para entender los problemas que afectan al mundo global. La participación 'saca' a la persona del ensimismamiento en el que vive y le hace ver más allá de sus propios problemas. Mediante la comprensión del funcionamiento de un barrio o ciudad y la participación activa en sus soluciones, el ciudadano comienza a entender mejor la complejidad

\footnotetext{
${ }^{7}$ Si bien actualmente ya se afirma que el beneficio económico no es separable del social o ambiental El fin de la empresa no debe ser únicamente el económico. Éste puede ser su motor, pero el objetivo debe ser producir beneficios en todos los sentidos (también sociales y ambientales) (García-Marzá, 2007).
} 
del mundo actual y está más preparado para hacer su aportación individual.

Refuerzo de la identidad. Donde existe una verdadera participación, se refuerza el sentimiento del ciudadano de pertenencia a un sitio determinado: su identidad. Ello redunda en un beneficio para el medio ambiente, pues una persona que se siente realmente formando parte de un lugar, entiende que su papel es importante, y tiende a cuidarlo. Así, la participación propicia que el habitante recupere su condición de ciudadano como «alguien preocupado de la cosa pública más allá de la votación periódica o de la afiliación a una u otra asociación» (Hernández, 2003).

Hasta aquí se han expuesto algunos requisitos previos que se deben cumplir. Pero, ¿en qué puntos del engranaje administrativo se puede aumentar la intervención ética?

Habitualmente se definen los siguientes niveles de planificación ambiental principales: a) políticas, planes y programas de gestión ambiental; b) evaluación ambiental de proyectos; c) gerencia ambiental de empresas; d) evaluación ambiental de productos. Los instrumentos por los que se evalúa la inclusión de la dimensión ambiental en los respectivos niveles son principalmente: la Evaluación Ambiental Estratégica (en políticas, planes y programas), la Evaluación de Impacto Ambiental (respecto a proyectos), los Sistemas Normalizados de Gestión (en la actividad empresarial general) y los etiquetados de productos (Gómez Orea et al., 2011).

En España, por ejemplo, algunos sectores han empezado a incorporar la ética ambiental de forma modesta pero progresiva. Así, en el mundo empresarial existen ya experiencias de gestión ética a través de la Responsabilidad Social Corporativa (que incluye la responsabilidad ambiental). Esto se realiza mediante códigos éticos, informes y auditorías éticas, así como comités de ética; en este último caso formados tanto por miembros de la corporación como por interlocutores sociales externos a la empresa (asociaciones de vecinos, ecologistas, etc.). Hay que señalar que estos sistemas benefician los intereses económicos de la empresa, pues sirven como publicidad, mejorando su legitimidad y credibilidad de cara a la opinión pública (García-Marzá, 2007).

Otro medio de participación ética sería el consumo responsable, que en parte es facilitado por los sistemas de 'etiquetado ecológico' o el etiquetado de la huella de carbono, de forma que el consumidor pueda saber qué productos tienen un mayor impacto sobre el medio ambiente y ejercer su responsabilidad moral a través del consumo (Bird, 2013).

A otros niveles, las consideraciones éticas en la planificación y gestión ambiental en general no han comenzado a incorporarse demasiado, si bien existen experiencias de consulta que incluyen a distintos actores sociales relacionados con la problemática ambiental, de forma que se han ido ampliando las posibilidades de participación ${ }^{8}$. Pero hay que avanzar más allá de la consulta social: es necesario extender los horizontes éticos con distintas perspectivas de análisis e incorporarlos progresivamente en las políticas ambientales y posteriormente en su ejecución a través de la planificación y gestión ambiental, así como en la elaboración de proyectos concretos.

\footnotetext{
8 Por poner tan sólo el ejemplo de Aragón en España, órganos tal que el Consejo de Protección de la Naturaleza en temas ambientales generales, incluyendo las áreas protegidas (http://www.aragon.es/DepartamentosOrganismosPublicos/Departamentos/DesarrolloRuralSostenibilidad/Consejo_Proteccion_Naturaleza_Aragon), o la Comisión del Agua en temas específicos sobre agua (http://www.aragon.es/DepartamentosOrganismosPublicos/Organismos/InstitutoAragonesAgua).
} 


\section{CONSIDERACIONES FINALES}

El desafío actual sigue siendo el modo en que se produce la incorporación de la ética en la gestión del medio ambiente. Tradicionalmente, la perspectiva antropocéntrica ha sido la dominante en la toma de decisiones en casi cualquier ámbito, a menudo con un carácter utilitarista. Como ya se ha indicado, desde hace unas décadas algunos autores abogan por un cambio de paradigma que, a partir del reconocimiento del valor intrínseco de la naturaleza, proponga cambios normativos sustanciales. No obstante, la problemática ambiental no entiende de fórmulas mágicas y resulta difícil creer que la solución consista simplemente en sustituir el paradigma antropocéntrico por el bio-ecocéntrico (Kwiatkowska, 2010).

Ambos pueden hacer aportaciones constructivas para la resolución de los problemas ambientales. De hecho, generalmente los especialistas en ética ambiental muestran un remarcable consenso en un gran número de cuestiones normativas y prácticas, aunque provengan de escuelas de pensamiento distintas, de forma que pueden hacer propuestas comunes para la planificación y gestión ambiental susceptibles de aceptación. Es decir, es posible alcanzar un consenso práctico, aunque no exista un consenso teórico total (Yang, 2010).

De esta forma, otro de los puntos de partida para una mayor inclusión de la ética en la toma de decisiones respecto a la planificación y gestión ambiental, sería incorporar comités de ética ambiental, consejos asesores, auditorías éticas o fórmulas de otro tipo en cada uno de los niveles.

Es fundamental añadir criterios éticos a las evaluaciones económicas y científico-técnicas habituales en planes y proyectos, además de asegurar que las políticas ambientales están exentas de cualquier tendencia o interés partidista. Los comités éticos velarían entonces por que las soluciones y medidas tomadas en cada caso se hayan logrado a partir de un amplio consenso y sean las mejores posibles para todos los actores involucrados, siempre incorporando en el debate todas las perspectivas de análisis posibles y no sólo las de carácter utilitarista, y finalmente certificando que el papel del partido en el gobierno es únicamente el de un gestor de la voluntad ciudadana ${ }^{9}$.

Quizás donde más claramente se ve la necesidad de una mayor presencia de la ética es en el nivel de las políticas ambientales: los objetivos y el escenario que se desean alcanzar con ellas deben analizarse también con criterios éticos. Lo mismo ha de suceder en los planes y programas de gestión ambiental. En ellos además es preciso incorporar nuevas perspectivas en las fases de detección de problemas y diagnóstico, así como en la definición de los fines que se persiguen con cada plan y programa concreto, de la misma forma que en las políticas. Igualmente, la propuesta de medidas concretas incluidas en cada plan y programa debe analizarse con la lupa de la ética ambiental, además de contar con una evaluación de su idoneidad y de la legitimidad de los medios que se utilizarán para llevarlas a cabo.

La evaluación ambiental de proyectos podría presentar ciertas dificultades respecto a la inclusión de las diversas perspectivas éticas, pues esto probablemente implicaría repensar y reconsiderar los actuales métodos de valoración y prevención de impactos (tanto directamente sobre la naturaleza a nivel de organismos individuales o de ecosistemas, como indirectamente sobre el medio ambiente humano). Nuevamente, se requiere también una reflexión profunda acerca de la conveniencia de las medidas preventivas, correctoras o compensatorias en cada caso, no sólo desde puntos de vista científico-técnicos o económicos, sino también con criterios éticos.

9 Pueden consultarse más propuestas para las políticas internacionales (no sólo sobre planificación ambiental) en Yang, Tongjin (2010). 
Finalmente, respecto a los niveles inferiores, por un lado se debe continuar trabajando en la expansión de las iniciativas que ya existen sobre gestión ética de la responsabilidad ambiental empresarial y buscar sinergias con la administración y organizaciones no gubernamentales, compartiendo conocimientos, experiencias y resultados; $y$, en cuanto al etiquetado de los productos, es imprescindible facilitar a los consumidores toda la información que sea posible acerca de la huella ecológica que provocan los bienes y servicios que adquieren, combinando esta tarea con un mayor esfuerzo en educación ambiental sobre consumo.

En definitiva, esta ampliación del marco de análisis ético sin duda contribuirá a reforzar los objetivos de conservación de la naturaleza, la lucha contra el cambio climático y en general la crisis ambiental actual, al tiempo que ayudará a evitar o minimizar los conflictos socioambientales relacionados.

\section{REFERENCIAS}

ALBA, J. Espacios naturales y desarrollo. En: D. M. Rivas (Coord.), Sustentabilidad. Desarrollo Económico, Medio Ambiente y Biodiversidad (p. 188-192). Madrid: Ed. Parteluz, 1997.

AMEND, T. \& AMEND, S. (Eds.). ¿Espacios sin habitantes?: parques nacionales de América del Sur. Caracas: UICN/Ed. Nueva Sociedad, 1992.

ARROJO, P., CASAUÚS, L. \& COPITZY, A. La rebelión de la montaña. Los conflictos del agua en Aragón. Bilbao: Bakeaz/FNCA, 2010.

BIRD, K. Calculating the carbon footprint of products - a quest for clarity. ISO News, 2013 (http://www.iso.org/iso/home/news_index/news_archive/news.htm?refid=Ref1801).

CARCAVILLA, L., LÓPEZ, J., DURÁN, J. J. Patrimonio geológico y geodiversidad: investigación, conservación, gestión y relación con los espacios naturales protegidos. Serie: Cuadernos del Museo Geominero, n. 7. Madrid: IGME, 2007.

CHUVIECO, E. Los Estados se comprometen a cuidar la "casa común». Palabra, n. 634, p. 8-12, 2016.

CHUVIECO, E. \& MARTÍN, M. A. Cuidar la Tierra: razones para conservar la Naturaleza. Madrid: Editorial Palabra/Digital Reasons, 2015.

ELCACHO, J. Los líderes budistas también reclaman un acuerdo contra el cambio climático. La Vanguardia, 2015 (http://www.lavanguardia.com/natural/20151030/54438468358/lideres-budistas-tambien-reclaman-un-acuerdo-mundial-contra-el-cambio-climatico.html).

ENTRALGO, J. R. Alcance y limitaciones de los sistemas urbanos de saneamiento. Situación de Zaragoza. Zaragoza: Ayuntamiento de Zaragoza, 2011 (https://www.zaragoza.es/contenidos/medioambiente/materialesdidacticos/calidad/JoseRamonEntralgoPresentacion.pdf).

GARCÍA-MARZÁ, D. Responsabilidad social de la empresa: una aproximación desde la ética empresarial. Veritas, v. II, n.17, p. 183-204, 2007.

GÓMEZ-OREA, D., GÓMEZ-VILLARINO, A. \& GÓMEZ-VILLARINO, M. T. EI Paisaje: Análisis, diagnóstico y metodología para insertarlo en la formulación de Planes y Proyectos. Madrid: AGV/Lulu, 2011.

HERNÁNDEZ, A. Ciudadanos fecundos: participación y calidad de vida. En: T. Arenillas (Coord.), Ecología y ciudad. Raíces de nuestros males y modos de tratarlos. Barcelona: El viejo Topo, 2003.

KWIATKOWSKA, T. ¡Que perdure la tierra! Poner en práctica la ética ambiental. En: H. Ten Have (Ed.), Ética ambiental y políticas internacionales (p. 175-202). París: UNESCO, 2010. 
LLAMAS, M. R., HERNÁNDEZ-MORA, N. \& MARTÍNEZ-CORTINA, L. El uso sostenible de las aguas subterráneas. Papeles del Proyecto Aguas Subterráneas, n.1. Santander: Fundación Botín, 2000.

MARCOS, A. Ética Ambiental. Valladolid: Universidad de Valladolid, 2001.

MATEO, J. M. 2003. Los caminos para el cambio. La incorporación de la sustentabilidad ambiental al proceso de desarrollo. La Habana: Editorial Científico-Técnica, 2003.

, J. M. Planificación Ambiental. La Habana: Editorial Félix Varela, 2008.

OLLERO, A. Guía metodológica sobre buenas prácticas en gestión de inundaciones. Manual para gestores. Zaragoza: Universidad de Zaragoza/Fundación Ecología y Desarrollo, 2014.

PERERO VAN HOVE, E. Participación ciudadana. Conclusiones del VI Congreso Nacional de Medio Ambiente: “Desarrollo Sostenible en España”. Madrid: Fundación CONAMA, 2002.

PIGEM, J. El mito del Cosmos desencantado. En: A. López, \& M. Tabuyo (Eds.), La naturaleza y el espíritu (p. 113-145). Palma de Mallorca, Ed. J.J. de Olañeta, 2006.

RODRÍGUEZ, A. Efectos de las carreteras sobre la fauna: un enfoque ecológico. En: VV.AA., Fauna y carreteras: el problema de la fauna en el proyecto, construcción y explotación de carreteras (p. 520). Madrid: Asociación Técnica de Carreteras/PIARC, 1999.

TEN HAVE, H. Introducción: medio ambiente, ética y políticas. En: H. Ten Have (Ed.), Ética ambiental y políticas internacionales (p. 11-23). París: UNESCO, 2010.

VELAYOS, C. La dimensión moral del ambiente natural: ¿necesitamos una nueva ética? Granada: Ed. Comares, 1996.

YANG, T. Hacia una ética ambiental global igualitaria. En: H. Ten Have (Ed.), Ética ambiental y políticas internacionales (p. 25-49). París: UNESCO, 2010. 